\title{
A Comparative analysis of OFDM based Communication using different Mapping Schemes for Signal Modulation
}

\author{
Nasir Mahmood, Muhammad Khalil Shahid, Ali Saeed \\ Senior Engineer PTCL, PTCL-ICT Islamabad \\ nasir_mahmood@ptcl.net.pk \\ Associate Professor, University of Lahore, Islamabad Campus \\ khalildona@gmail.com \\ Research Associate, PTCL-ICT Islamabad \\ alisaeed01@yahoo.com
}

\begin{abstract}
OFDM is a multiplexing technique that divided a spectrum into sub-carriers that are orthogonal to each other. They are capable to carry high rate data transmission. The orthogonality is maintained as one sub carrier is null on the centre of other sub carrier. Sub-carriers have cyclic prefix is put in between and bandwidth wastage due to guard band, as in case of FDMA, is reduced up to $50 \%$ in most of the cases. Spectral efficiency is of OFDM is far more better than other frequency modulation techniques due to othrognality and CP design that helps in better diversity gain. The data is dispensed as sub-streams among sub carriers and each of the sub carrier is modulated as per the coded (PSK, BPSK, QAM) data sub-streams.
\end{abstract}

Thus rather modulating whole data stream over a single frequency, sub carriers are allocated portions of data that ensure large amount of data processing. To ensure orthogonality, serial data is converted to parallel streams and are fed to IFFT module, from where P/S conversion is performed and CP is inserted between two sub-carriers. The reverse of these steps happens in the receiving module. Important aspects of OFDM systems are, Synchronization, Pilot allocation, the Channel State Information or Channel estimation etc. However this paper takes up channel estimation techniques (channel state information) in OFDM systems and puts a comparative analysis of them. It will help in understanding merits and demerits of estimation techniques that are in use.

\section{Indexing terms/Keywords}

IFFT, FFT, OFDM, Modulation, Multiplexing.

\section{Academic Discipline And Sub-Disciplines}

Telecommunication, Comunication Sciences, Mathematics

\section{SUBJECT CLASSIFICATION}

Wireless Communication (OFDM)

\section{TYPE (METHOD/APPROACH)}

\section{Simulation}

\section{Council for Innovative Research}

Peer Review Research Publishing System

\author{
Journal: INTERNATIONAL JOURNAL OF COMPUTERS \& TECHNOLOGY
}

Vol 12, No. 10

editor@cirworld.com 


\section{INTRODUCTION}

Signal through various paths, from transmitter, reaches the receiver. This propagation of signal from multiple paths impairs each signal in varying ways. Many impairments that causes signal to lose SNR and its intactness include ISI, ICI, Small scale fading, larges scale fading etc. Noise addition is another important aspect of signal distortion. This distortion, along with restriction to high data rate transmission due to limited frequency usage, had hampered new generation data services.

It is therefore easy to comprehend that if the data is dispensed among different subcarriers, high data rates and be achieved and impairments to single carrier can be mitigated.

For the purpose of distinguishing among subcarriers that carry divided data, two multiplexing techniques have gained great importance [2].

i. $\quad$ FDM (Frequency Division Multiplexing)

ii. $\quad$ CDM (Code Division Multiplexing)

In first methodology frequency is divided into subcarriers and thus distinction is achieved through frequency differences. However another way is to distinguish among carriers is through codes in which separate codes are allocated for multiplexing. Another way, which is more efficient and saves bandwidth, is OFDM [4].

OFDMA is an access technology that makes use of OFDM which divides a given spectrum into number of sub carriers, which are orthogonal to each other and thus produce higher spectral efficiency and save great deal of bandwidth as shown in Fig.1
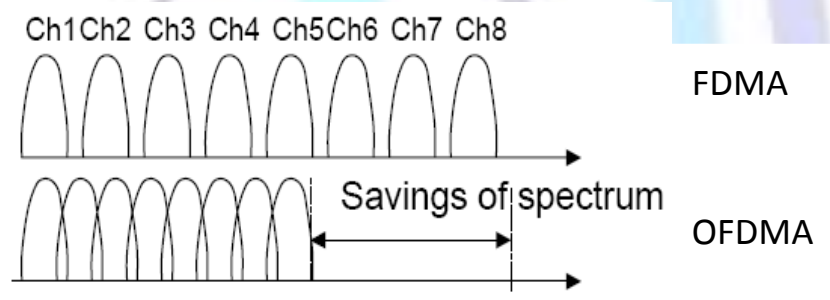

Fig1. Comparison of OFDM and FDM

Fig 2 shows a spectrum which is divided into $\mathrm{N}$ number of subcarriers for the purpose of OFDM. Data is dispensed to each of the subcarrier and this each carrier is modulated using a scheme like PSK, QAM etc. usually N number of carriers are not less than 256, and converted into time domain (as per OFDM modem design to retain othogonality and sent to upconverter for transmission. The reverse of all that happened at the transmitter station happens and the signal is converted into frequency domain. After complete reception each carrier is demodulated for data retrieval [6]. Before the up conversion and transmission, as told above, two subsequent carriers are inserted with Cyclic Prefix in between them.

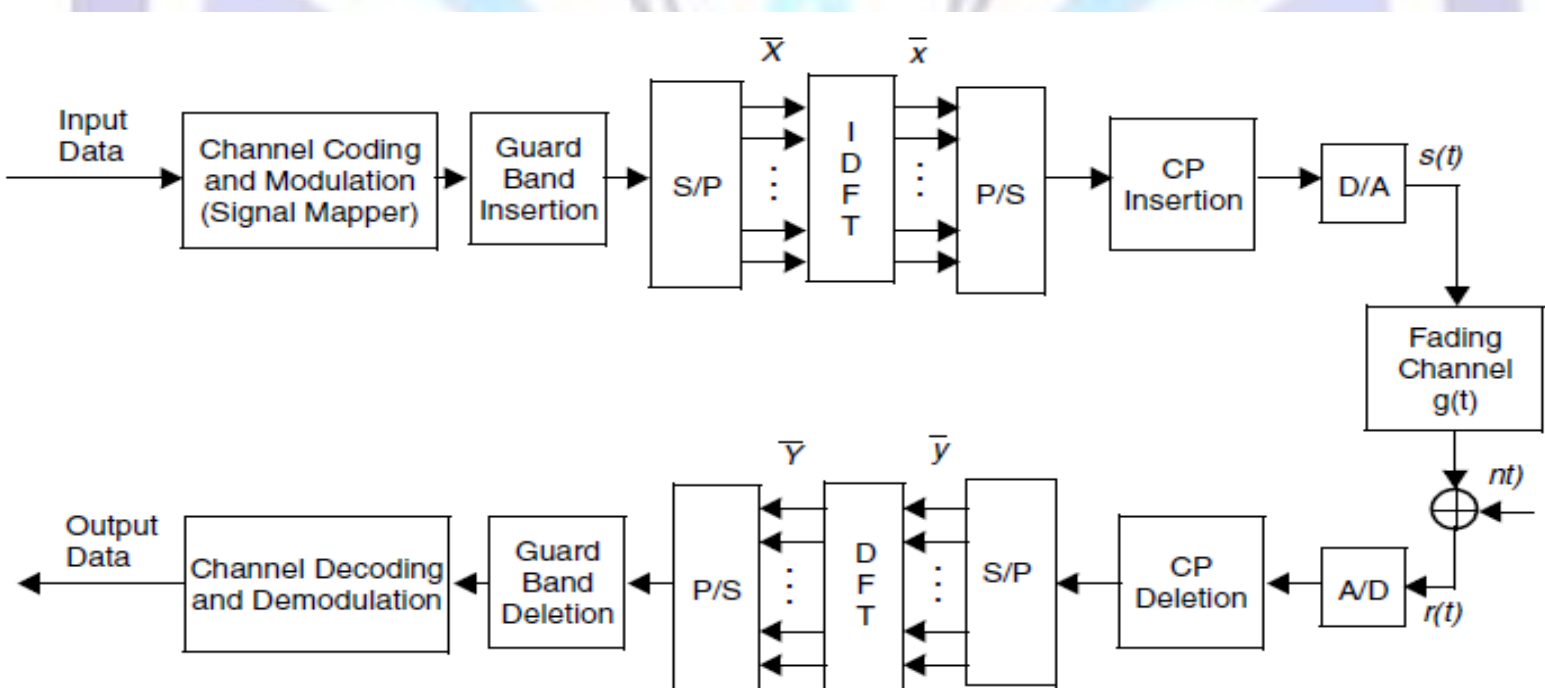

After simplified explanation of the OFDM Modulation and Demodulation, mathematical representation of the system is important in order to understand OFDM system in detail. When a symbol is mapped on a carrier frequency, it is grouped into column vector so that it might be fed to IFFT module (S/P conversion, shown in Fig 2) the entries of the column matrix are the same as the size of FFT: 


$$
\vec{S}(m)=\left[\begin{array}{c}
S(m N) \\
S(m N+1) \\
\cdot \\
\cdot \\
\cdot \\
S(m N+N-1)
\end{array}\right]_{N \times \mathbf{1}} \ldots \ldots \ldots(\mathbf{1})
$$

Whereas: $\vec{S}(m)$ is $\mathrm{m}$-th Symbol

\section{Simplified Block and Mathematical Model of OFDM System:}

Refer to the fig.2. Channel coding is applied on data for reliable transmission. Then this data is interleaved to overcome the burst error. Then data is mapped by using BPSK, QPSK or QAM. Then these frequency domain modulated symbols are arranged in parallel pattern, by applying BPSK, QPSK or QAM and then converted into time domain symbols by using IFFT algorithm. In the above mentioned matrix equation and OFDM block diagram

$\mathrm{N}=$ size of IFFT

$\mathrm{m}=$ Index of Symbol

As shown in Fig 2 the F/T conversion by IFFT module can be represented as below which mathematically represents the system:

$$
\begin{aligned}
\vec{s}(m) & =\left[\begin{array}{c}
s(m N) \\
s(m N+1) \\
\cdot \\
\cdot \\
\dot{v} \\
s(m N+N-1)
\end{array}\right]_{N \times 1} \\
& =\left(\frac{1}{N}\right) F_{N}^{H} \vec{S}(m)
\end{aligned}
$$

Whereas, $\vec{s}(m)$ is time domain vector

$\mathrm{F}_{\mathrm{N}}=\mathrm{N}-\mathrm{FFT}$ matrix

Cyclic prefix is appended after the carrier and also is pre-pended, in order to avoid cross talk between two sub carriers. Mathematically the presence of CP can be included in the above mentioned symbol's vectors are:

Let $\vec{u}(m)$ be the vector matrix of the CP that is appended to the carriers, then:

$$
\vec{u}(m)=\left[\begin{array}{c}
u\left(m N_{\text {tot }}\right) \\
u\left(m N_{\text {tot }}+1\right) \\
\cdot \\
\cdot \\
\cdot \\
\cdot \\
u\left(m N_{\text {tot }}+N_{\text {tot }}-1\right)
\end{array}\right]_{N_{\text {tot }} \times 1}
$$

Whereas: $\mathrm{N}_{\text {tot }}=\mathrm{N}_{\mathrm{g}}+\mathrm{N}$

It can be written in mathematical form that, $\vec{u}(\mathrm{~m})$ : 


$$
\vec{u}(m)=A_{c p} \vec{s}(m)
$$

Where,

$$
A_{c p}=\left[\begin{array}{cc}
0 & I_{N_{g}} \\
I_{N-N_{g}} & 0 \\
0 & I_{N_{g}}
\end{array}\right]_{\left(N+N_{g}\right) \times N}
$$

$\mathrm{N}_{\mathrm{g}}$ which should be greater than $\mathrm{L}$ in order to eliminate delay spread i.e. $\mathrm{N}_{\mathrm{g}} \geq \mathrm{L}$. Insertion of Cyclic Prefix the delay spread can be avoided thus mitigating ICI and ISI in transmission. The value of CP is taken as 16 as per IEEE 802.11a standards [4].

The cumulative signal, as shown in both the vectoral representation (symbol and cyclic prefix), is sent to DAC which then transmit the signal through RF arrangement. This signal attaining amplitude, frequency distortion and noise is received at the other end (as shown in fig 2). In our case to keep things simple and for comparison purpose, Quasi Static -Selective Rayleigh fading channel has been used [7]. Also that, the channel has be considered constant so that parameters related to channel estimation/ channel state information might not complex this research. The channel is considered as (L1)-th order FIR with filter co-efficient $\left\{h_{0}, h_{1}, h_{2} \ldots h_{1} \ldots \ldots . h_{L-1}\right\}$ as shown in fig 4.2

The channel impulse response of this channel for $\mathrm{m}$-th Symbol can be stated in matrix form as:

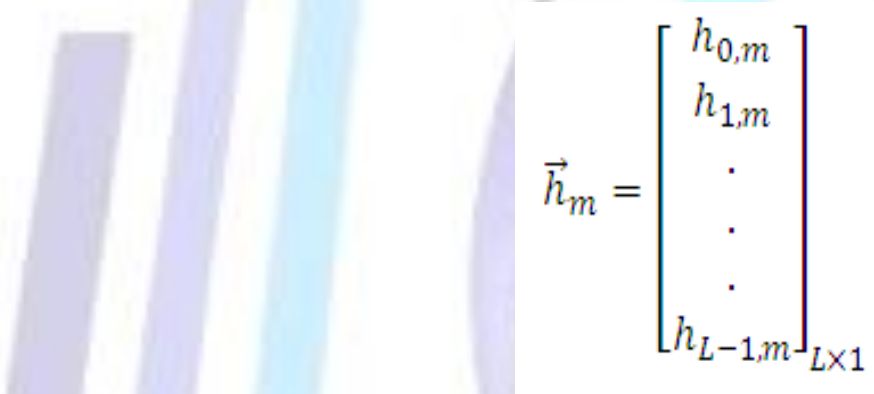

Subscription, $m$ denotes different value of Channel Impulse Response for each of the OFDM symbol.

The overall baseband mathematical model for the received can be obtained by addition of AWGN to the result of convolution of ' $U$ ' and ' $h$ ' vectors that represent the CP + Symbol and Channel Impulse response respectively. The sample of OFDM n-th in number can be represented as:

$$
\begin{aligned}
& r\left(m N_{\text {tot }}+n\right)=\sum_{l=0}^{L-1} h_{l, m} \vec{u}\left(m N_{\text {tot }}+n-l\right)+ \\
& \vec{v}\left(m N_{\text {tot }}+n\right) \ldots \ldots \ldots \ldots \ldots \ldots \ldots \ldots \ldots \ldots \ldots \ldots \ldots
\end{aligned}
$$

Whereas,

\section{$0 \leq \mathrm{n} \leq$ Ntot -1}

$$
\begin{aligned}
& r\left(m N_{\text {tot }}+n\right)=\text { n-th sample received during } m \text {-th symbol reception at OFDM receiving end. } \\
& v\left(m N_{\text {cot }}+n\right)=A W G N \text { at }\left(m_{\text {ton }}+n\right) \text { th sample. }
\end{aligned}
$$

Since the above expression represents last sample of the OFDM subcarrier, it can be used to present the whole signals transfer to the receiver end as given below [3]. 


$$
\begin{aligned}
& \vec{r}(m)=\left[\begin{array}{c}
r\left(m N_{\text {tot }}\right) \\
r\left(m N_{\text {tot }}+1\right) \\
\cdot \\
\cdot \\
\cdot \\
\cdot \\
r\left(m N_{t o t}+N_{\text {tot }}-1\right)
\end{array}\right]_{N_{\text {tot }} \times 1} \\
& \vec{v}(m)=\left[\begin{array}{c}
v\left(m N_{\text {tot }}\right) \\
v\left(m N_{\text {tot }}+1\right) \\
\cdot \\
\cdot \\
\cdot \\
\cdot \\
v\left(m N_{\text {tot }}+N_{\text {tot }}-1\right)
\end{array}\right]_{N_{\text {tot }} \times 1} \\
& \boldsymbol{h}_{\text {m, Toep }}=\left[\begin{array}{ccccccc}
h_{0, m} & 0 & 0 & 0 & \cdot & \cdot & 0 \\
h_{1, m} & h_{0, m} & 0 & 0 & \cdot & \cdot & 0 \\
\cdot & h_{1, m} & h_{0, m} & 0 & \cdot & \cdot & 0 \\
\cdot & \cdot & h_{1, m} & h_{0, m} \cdot & \cdot & 0 \\
\cdot & \cdot & \cdot & h_{1, m} \cdot & \cdot & 0 \\
h_{L-1, m} & \cdot & \cdot & \cdot & \cdot & \cdot & 0 \\
0 & h_{L-1, m} & \cdot & \cdot & \cdot & \cdot & 0 \\
0 & 0 & h_{L-1, m} & \cdot & \cdot & \cdot & 0 \\
0 & 0 & 0 & h_{L-1, m} & \cdot & h_{0, m}
\end{array}\right] \\
& \boldsymbol{h}_{m, \text { Toep }}^{(c)}=\left[\begin{array}{ccccccc}
h_{0, m} & 0 & 0 & 0 & \cdot & \cdot & 0 \\
h_{1, m} & h_{0, m} & 0 & 0 & \cdot & \cdot & 0 \\
\cdot & h_{1, m} & h_{0, m} & 0 & \cdot & \cdot & 0 \\
\cdot & \cdot & h_{1, m} & h_{0, m} & \cdot & \cdot & 0 \\
\cdot & \cdot & \cdot & h_{1, m} & \cdot & \cdot & 0 \\
h_{L-1, m} & \cdot & \cdot & \cdot & \cdot & \cdot & 0 \\
0 & h_{L-1, m} & \cdot & \cdot & \cdot & \cdot & 0 \\
0 & 0 & h_{L-1, m} & \cdot & \cdot & \cdot & 0 \\
0 & 0 & 0 & h_{L-1, m} & \cdot & h_{0, m}
\end{array}\right]
\end{aligned}
$$

$h_{m, T o e p}$ is Troeplitz-matrix for Channel Impulse Response vector in case of $\mathrm{m}$-th symbol.

DT (discrete time) convolution can be taken by using ' $h$ ' and ' $U$ ' vector which for received signal of $m$-th position in OFDM symbol will be:

$$
\vec{r}(m)=h_{m, T o E p} \vec{u}(m)+h_{m, T 0 E p}^{(c)} \vec{u}(m-1)+\vec{v}(m)
$$

$\mathrm{v}(\mathrm{m})$ is the $A W G N$ and term

$$
\boldsymbol{h}_{m, T \circ \theta p}^{(c)} \vec{u}(m-1)
$$

represents ISI and also that ISI will effect $L-1$ terms.

Let $y(m)$ be the output after removing CP. ISI will be mitigated if the condition that delay spread $\mathrm{Ng}$ is less than L. $y(\mathrm{~m})$ can be represented as 


$$
\vec{y}(m)=\left[\begin{array}{c}
y(m N) \\
y(m N+1) \\
\cdot \\
\cdot \\
\cdot \\
y(m N+N-1)
\end{array}\right]_{N \times 1}
$$

$\vec{y}(m)$ can also be expressed in matrix form as:

$$
\vec{y}(m)=A_{C P R} \vec{r}(m)
$$

Where,

$$
A_{C P R}=\left[\begin{array}{ll}
0 & I_{N}
\end{array}\right]_{N \times N_{\text {tot }}}
$$

The signal after transformation from FFT is in time domain and can be represented as:

$$
\vec{Y}(m)=\left[\begin{array}{c}
Y(m N) \\
Y(m N+1) \\
\cdot \\
\cdot \\
\cdot \\
\cdot \\
Y(m N+N-1)
\end{array}\right]_{N \times 1}
$$

This Equation can be written in matrix form by using FFT matrix $\boldsymbol{F}_{N}$

$$
\begin{aligned}
& \vec{Y}(m)= \boldsymbol{F}_{N} \vec{y}(m) \\
&= \boldsymbol{F}_{N} \boldsymbol{A}_{C P R} \vec{r}(m) \\
&= \boldsymbol{F}_{N}\left\{\boldsymbol { A } _ { C P R } \left[\boldsymbol{h}_{m, \text { Toep }} \vec{u}(m)\right.\right. \\
&\left.\left.\quad+\boldsymbol{h}_{m, \text { Toep }}^{(c)} \vec{u}(m-1)+\vec{v}(m)\right]\right\} \\
&=\boldsymbol{F}_{N}\left\{\boldsymbol{A}_{C P R} \boldsymbol{h}_{m, \text { Toep }} \vec{u}(m)+\boldsymbol{A}_{C P R} \vec{v}(m)\right\} \\
&=\boldsymbol{F}_{N}\left\{A_{C P R} \boldsymbol{h}_{m, \text { Toep }} \boldsymbol{A}_{c p} \vec{s}(m)+A_{C P R} \vec{v}(m)\right\} \\
&=\boldsymbol{F}_{N}\left\{A_{C P R} \boldsymbol{h}_{m, T o e p} \boldsymbol{A}_{c \boldsymbol{p}}\left(\frac{1}{N}\right) \boldsymbol{F}_{N}^{H} \vec{S}(m)+\right. \\
&\left.\quad A_{C P R} \vec{v}(m)\right\} \\
&=\frac{1}{N} \boldsymbol{F}_{N}\left\{A_{C P R} \boldsymbol{h}_{m, T o e p} A_{c p}\right\} \boldsymbol{F}_{N}^{H} \vec{S}(m) \\
& \quad+\boldsymbol{F}_{N}\left(A_{C P R} \vec{v}(m)\right)
\end{aligned}
$$




$$
\begin{aligned}
=\frac{1}{N} \boldsymbol{F}_{N}\left\{A_{C P R} \boldsymbol{h}_{m, T o e p} A_{c p}\right\} \boldsymbol{F}_{N}^{H} \vec{S}(m) \\
+\boldsymbol{F}_{N}\left(A_{C P R} \vec{v}(m)\right) \\
=\frac{1}{N} \boldsymbol{F}_{N}\left\{A_{C P R} \boldsymbol{h}_{m, \text { Toep }} A_{c p}\right\} \boldsymbol{F}_{N}^{H} \vec{S}(m) \\
\quad+\boldsymbol{F}_{N}\left(A_{C P R} \vec{v}(m)\right) \\
=\frac{1}{N} \boldsymbol{F}_{N}\left\{\boldsymbol{h}_{m, \text { Cir }}\right\} F_{N}^{H} \vec{S}(m)+\vec{V}(m) \ldots . .
\end{aligned}
$$

$$
\begin{aligned}
& \text { Where } \vec{V}(m)=F_{N}\left(A_{C P R} \vec{v}(m)\right) \text { and } \boldsymbol{h}_{m, \text { Cir }}= \\
& A_{C P R} \boldsymbol{h}_{m, \text { Toep }} A_{c p}
\end{aligned}
$$

$\boldsymbol{h}_{\text {Cir }}$, is circular matrix of order $\mathrm{N} \times \mathrm{N}$. And thus circulant matrix can be represented as:

$$
\left[\begin{array}{c}
h_{0, m} \\
h_{1, m} \\
\cdot \\
\cdot \\
h_{L-1, m} \\
0_{(N-L) \times 1}
\end{array}\right]_{N \times 1}
$$

Important aspects of circulat matrix [38] are given as:

- Eigen vectors of circular matrix $\mathrm{N} \times \mathrm{N}$ are columns of $\mathrm{N}$ - point inverse fast Fourier transform namely $\boldsymbol{F}_{N}^{H}$

- The single entry of circulant-matrix is the FFT of first-column. Decomposition of matrix may be performed as $\boldsymbol{F}_{N}^{H} \Delta \boldsymbol{F}_{N}$, where delta is the diagonal matrix, with Eigen values in diagonal

Therefore Eigen values of $\boldsymbol{h}_{m-c}$ are:

Where $\mathrm{C}=$ Channel Impulse response and is also shown as $\mathrm{CIR}$

$$
\left[\begin{array}{c}
H_{0, m} \\
H_{1, m} \\
\cdot \\
\cdot \\
\cdot \\
H_{N-1, m}
\end{array}\right]_{N \times 1}=F_{N}\left[\begin{array}{c}
h_{0, m} \\
h_{1, m} \\
\cdot \\
\cdot \\
h_{L-1, m} \\
0_{(N-L) \times 1}
\end{array}\right]_{N \times 1}
$$

Decomposition of values that are Eigen Values will be: 


$$
\boldsymbol{h}_{m, \text { Cir }}=\frac{1}{N} \boldsymbol{F}_{N}^{H}\left[\begin{array}{ccc}
H_{0, m} & \cdots & 0 \\
\vdots & \ddots & \vdots \\
0 & \cdots & H_{N-1, m}
\end{array}\right] \boldsymbol{F}_{N} \ldots \ldots(\mathbf{9})
$$

Substituting this value in equation (8), we get the final expression as:

$$
\begin{gathered}
\vec{Y}(m)=\boldsymbol{H}_{m, \operatorname{diag}} \vec{S}(m)+\vec{V}(m) . \\
\text { Where, } H_{k, m}=\sum_{l=0}^{L-1} h_{l} e^{-j 2\left(\frac{\pi}{n}\right) k l} 0 \leq \mathrm{k} \leq \mathrm{N} .
\end{gathered}
$$

The above mentioned equation is the representation of OFDM signal. This mathematical representation is complete in explaining the OFDM transmitter and receiving process and helps in understanding equalization, channel estimation etc. These expressions have been used in MATLAB for the simulation purpose and a comparison by changing modulation techniques has been shown.

\section{Simulation}

\section{System Parameters}

The parameters and values that have been fixed in this simulation are given in Table. 1. In simulation no realistic channel has been used and the values of AWGN have been incorporated rather any other impairment to keep the results simple.

\begin{tabular}{|l|l|}
\hline Parameter & Value \\
\hline Channel & AWGN \\
\hline Mapping Scheme & BPSK,QPSK, 8QAM,16QAM \\
\hline FFT Size & 64 \\
\hline Number of subcarrier & 52 (For BPSK) \\
\hline No. of bits per OFDM symbol & 52 \\
\hline No. of Symbols & 10000 \\
\hline Pilot Ratio & N/A \\
\hline Sample Time & 10000 Second \\
\hline Doppler Shift & $10 \mathrm{~Hz}$ \\
\hline
\end{tabular}

Table1. OFDMA Parameters for Simulation

Different plot between BER and Eb/No or SNR have been obtained with the use of different mapping schemes. Cyclic Prefix is greater enough to cause any delay spread (cause of ISI). The number of bits per symbol of OFDM and the number of carriers are the same in all cases. 


\section{Simulation Results}

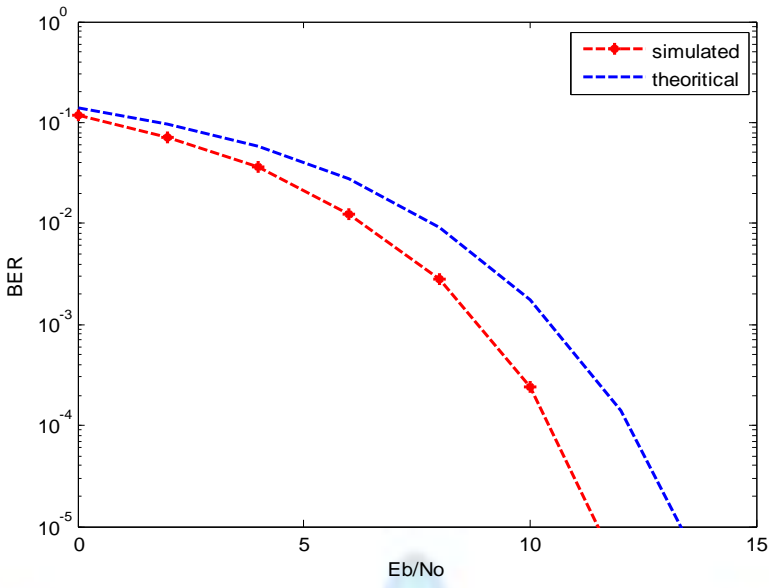

Fig.3 Bit Error Rate for OFDMA System using 8-QPSK

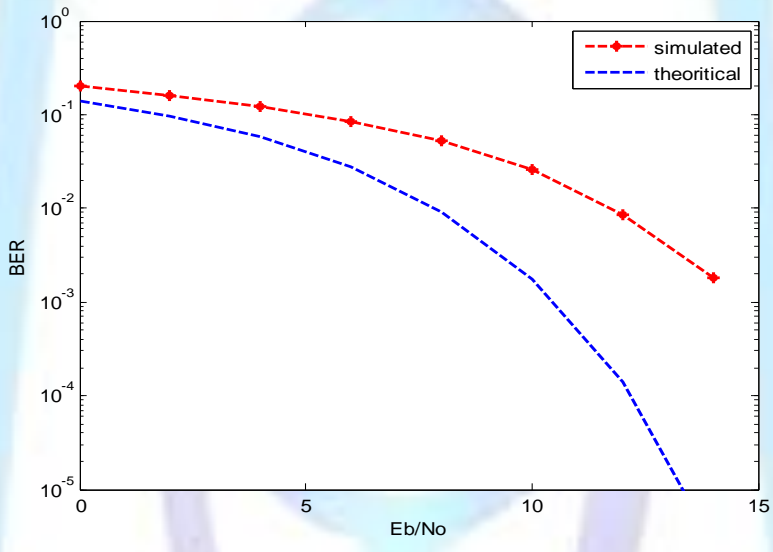

Fig.4. BER performance for OFDMA System using 16-QPSK

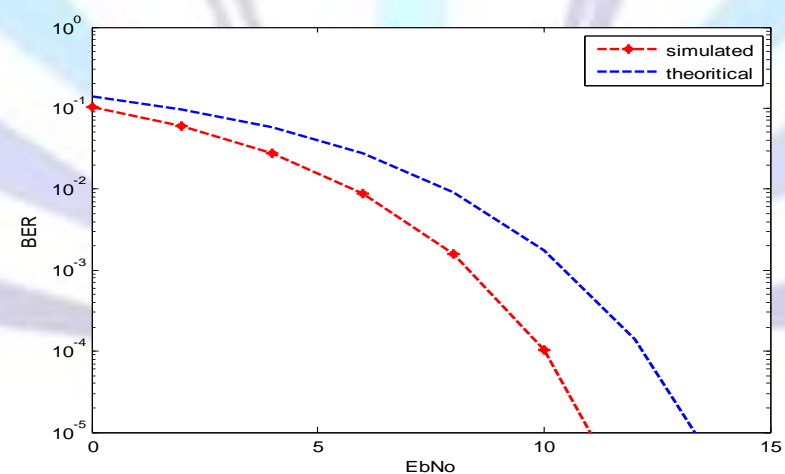

Fig.5. Bit Error Rate for OFDMA System using 8-QAM 


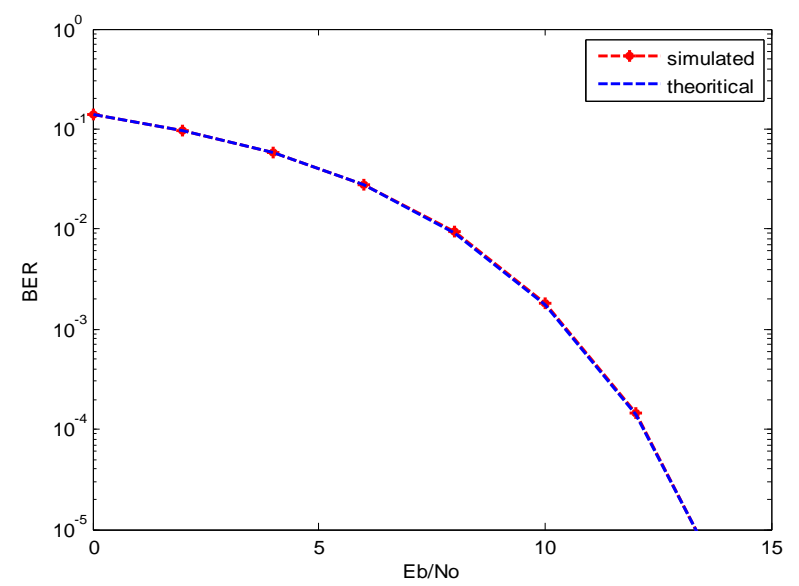

Fig.6. Bit Error Rate for OFDMA System using 16-QAM

\section{Conclusion}

The simulation has been performed in order to check the degradation in transmission, bit error rate for the same set of conditions for different modulation techniques. The channel conditions have been taken as ideal however simple AWGN impairments have been added. The simulation has shown that QPSK technique has performed better than any of the techniques used. It can also be concluded that the communication system that uses OFDM has higher bit error rates in case higher bandwidth efficient modulation schemes are used. This is because of using smaller spectrum to transfer high data rate and is significantly wastage of advantage that is provided by OFDM.

\section{ACKNOWLEDGMENTS}

Our thanks to the experts who have contributed towards development of the template.

\section{REFERENCES}

[1] Jeruchim, Balaban, and Shanmugan, "Simulation of Communication Systems",

[2] Proakes, "Digital Communications", 3rd edition, McGraw-Hill, 1995, 929 p.

[3] Moon, Sterling "Mathematical Model and Algorithm"

[4] M. Brookers, "Matrix Reference Manual [online]", available:

[5] http://www.ee.ic.ac.uk/hp/star/dmb/matrix/.

[6] Vahlin and Holte, "Optimal-duration pulses for OFDM," IEEE Transaction Communication., Jan. 1996.

[7] Harada, Prasad "Simulation and Software radio for Mobile Communications"

\section{Author' biography}

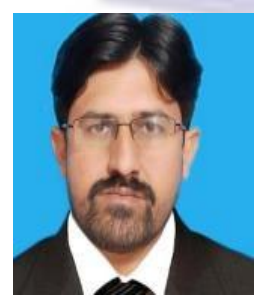

Nasir Mahmood received B.Sc. Engineering degrees in Electrical \& Electronic Engineering from Bangladesh Institute of Technology (BIT) Rajshahi in 2000. Thereafter he received PGD in Telecommunication from National University of Sciences and Technology (NUST) in 2002. He has served in Pakistan broadcasting corporation as senior broadcast engineer and then joined Pakistan Telecommunication Company limited (PTCL). These days he is working as Manager Training (Technical) at Institute of Communication Technologies(ICT). 